\title{
EVALUACIÓN DEL EFECTO SANITIZANTE DE UN EXTRACTO BIODEGRADABLE OBTENIDO DE LA ESPECIE Solanum marginatum, DE USO ETNOBOTÁNICO EN BOYACÁ
}

\author{
MAURICIO GARCÍA COLMENARES ${ }^{1}$ \\ MARÍA CRISTINA CASTELLANOS CORREDOR ${ }^{2}$
}

Manizales, 2010-09-01 (Rev. 2010-10-11)

\section{RESUMEN}

Las plantas del género Solanum, dentro del que se encuentra el "lulo de perro" (Solanum marginatum), especie considerada una maleza, contienen saponinas, las cuales tienen propiedades de emulsionar la grasa, similares al jabón. Esta característica fue aprovechada para evaluar el poder sanitizante del extracto alcohólico del fruto, en calidad de limpiador biodegradable, de gran utilidad en

industria.

El potencial sanitizante del limpiador preparado se evaluó según el procedimiento descrito por Carrascal, Páez y Burbano (1998), y se encontró como condiciones óptimas de efectividad antimicrobial: una concentración del $10 \%$ y un tiempo de contacto de 6 minutos sobre microorganismos bacilos gram negativos, que presentan un máximo de inhibición de 99,58\%. Al comparar la efectividad del producto obtenido contra la de dos conocidos sanitizantes comerciales (Mat-98® y Germigel $\left.{ }^{\circledR}\right)$, se encontró una respuesta similar.

Por hidrólisis del crudo de saponina se liberaron las sapogeninas presentes, los cristales obtenidos se analizaron por espectroscopía infrarroja y se les determinó el punto de fusión, coincidiendo los resultados con la Hecogenina.

PALABRAS CLAVE:

Solanum marginatum, saponinas, sapogeninas, sanitizante natural, desinfectante.

EVALUATION OF THE SANITIZER EFFECT OF A BIODEGRADABLE EXTRACT OF ETHNOBOTANICAL USE IN BOYACÁ, OBTAINED FROM THE Solanum marginatum SPECIES

\section{ABSTRACT}

Plants of the genus Solanum, within which the "Lulo perro" (Solanum marginatum) is found and is considered as a weed species, contain saponins which have fat emulsifying properties similar to soap. This feature was used to evaluate the sanitizing power of the alcoholic extract of the fruit as a biodegradable cleaner which is very useful for industrial purposes.

The sanitizing potential of the cleaner preparation was assessed using the procedure described by Carrascal, Páez y Burbano (1998), and it was found to have optimum antimicrobial effectiveness conditions: a 10\% concentration and a 6 minute contact time over gram negative bacilli microorganisms which represent a maximum inhibition of $9958 \%$. When comparing the effectiveness of the product against two well-known commercial sanitizers. (Mat-98® and Germigel () , a similar response was found.

Using crude hydrolysis of saponin, present sapogenins were released, the crystals obtained were analyzed using infrared spectroscopy, and melting point was determined. These results coincided with the Hecogenin.

KEY WORDS: solanum marginatum, saponins, sapogenins, natural sanitizer, disinfectant.

\section{INTRODUCCIÓN}

Las saponinas son glucósidos naturales ampliamente distribuidas en el reino vegetal y hasta el presente se las ha detectado en más de 500 géneros de plantas, como las Solanum, dentro de la que se encuentra el "lulo de perro" (Solanum marginatum), objeto del presente estudio. Se caracterizan por tener propiedades similares al jabón, al producir espuma cuando son agitados en 
agua (Fieser, 1959) y emulsionar la grasa; además de producir hemólisis de variada potencia, son tóxicas para animales de sangre fría e irritantes de la mucosa nasal y faríngea.

En algunas regiones de Latinoamérica, se ha reportado el uso etnobotánico de especies Solanum como agentes limpiadores. En Colombia se han realizado estudios sobre la composición del fruto, evaluando y caracterizando ciertos alcaloides (Herrera \& Kecán, 1970; Sanabria, 1974); sin embargo, no se han encontrado reportes sobre la evaluación de la especie "lulo de perro" como agente limpiador, la cual es utilizada tradicionalmente por las comunidades campesinas para tal fin, en la Provincia de Norte del departamento de Boyacá. Para evaluar el efecto sanitizante de esta planta, considerada una "maleza", se recolectaron los frutos silvestres, se lavaron, desinfectaron y secaron. El material seco fue triturado, homogenizado y tamizado, y se procedió a realizar la extracción de las saponinas.

El procedimiento para la extracción de saponinas de matrices vegetales ha sido evaluado y reportado en la bibliografía siguiendo múltiples rutas, pero dada la naturaleza ampliamente polar de estos compuestos, todos los métodos coinciden en la extracción en caliente o en frio, con agua o alcoholes de bajo peso molecular como metanol, etanol y butanol o mezclas acuosas de éstos en diversas proporciones (Martínez, 2001).

La extracción de las saponinas se inició con etanol al 70\% y se continuó hasta agotamiento, con nbutanol. Mediante ensayos cualitativos específicos sobre el extracto hidroalcohólico, se comprobó la presencia de saponinas, las cuales consisten en el principio activo que actúa como limpiador biodegradable, interés fundamental de este trabajo. Por hidrólisis de las saponinas se obtuvieron cristales de sapogenina, cuya estructura química, elucidada mediante espectroscopia infrarroja y punto de fusión, coincide con la hecogenina.

La evaluación del potencial sanitizante del extracto obtenido se hizo con la prueba estándar de desinfección (Carrascal, Páez \& Burbano, 2003), mediante la cual se cuantifica el porcentaje de inhibición de microorganismos (bacilos gram negativos). Los resultados fueron comparados con dos limpiadores comerciales de uso común en la industria alimentaria: Mat-98® y Germigel@.

\section{MATERIALES Y MÉTODOS}

\section{Recolección y preparación de la muestra}

Los frutos de Solanum marginatum, "lulo de perro", se recolectaron quincenalmente durante los meses de octubre, noviembre y febrero, de plantas crecidas en forma silvestre en la ciudad de Tunja. La pulpa del fruto se separó manualmente de la corteza y semillas, y se redujo a trozos de $0,5 \mathrm{~cm}$, las cuales se secaron en horno $\mathrm{MLW}$ a $45^{\circ} \mathrm{C}$ durante 9 días. El material seco fue triturado en molino mecánico (corona) y homogenizado a un tamaño de partícula de $100 \mu \mathrm{m}$ con la ayuda de un tamiz $17 \mathrm{~N}-100$; además, se mantuvo en desecador con silica gel a temperatura ambiente, hasta su procesamiento.

\section{Extracción del crudo de saponinas}

La pulpa seca se maceró en etanol al $70 \%$ durante 48 horas, siguiendo el procedimiento descrito por Wall (1952), y el extracto se filtró y reservó. Se repitió la extracción con n-butanol durante 24 horas. Se reunieron los extractos y se concentraron en rotaevaporador (BÛCHI R-205), el residuo se colocó en embudo de separación y se desengrasó con éter de petróleo, la fracción acuosa de disolvió en $40 \mathrm{ml}$ de agua destilada y se extrajo hasta máximo agotamiento con n-butanol con ayuda de embudo de separación, y se concentró en rotaevaporador hasta obtener un residuo pastoso de color rojo oscuro con olor acaramelado, correspondiente al crudo de saponinas.

\section{Identificación de saponinas}

La presencia de saponinas en el extracto hidroalcohólico concentrado se comprobó mediante ensayos químicos cualitativos: Espuma, Hemólisis, Liebermann-Burchard, Salkowski, Molish, Antrona, Rosemheim y Vainillina, todos con resultados positivos.

\section{Hidrólisis de saponinas}

La hidrólisis de saponinas se realizó siguiendo el procedimiento descrito por Segal, Mansour y Saitschek (1966), el cual consistió en disolver $2 \mathrm{~g}$ del crudo de saponinas en $50 \mathrm{ml}$ de etanol al 50 $\%$. A esta disolución se adicionaron $10 \mathrm{ml}$ de solución de $\mathrm{KOH} 1 \mathrm{~mol} / \mathrm{L}$ en metanol, y se mantuvieron en reflujo durante 2 horas. La solución se refrescó y acidificó ligeramente con solución de $\mathrm{HCl}$ 0,5 
$\mathrm{mol} / \mathrm{L}$ y se evaporó el solvente con corriente de aire a temperatura ambiente. El residuo se lavó dos veces con pequeños volúmenes de agua y se cristalizó en metanol.

\section{Asignación de estructura química}

El residuo obtenido de la hidrólisis de saponinas corresponde a cristales de sapogeninas, los cuales se identificaron por medio de espectroscopia infrarroja y punto de fusión. Los resultados se compararon con dos patrones (diosgenina y hecogenina) y se asignó la estructura química correspondiente.

\section{Evaluación del efecto sanitizante del extracto de saponinas}

El extracto hidroalcohólico de saponinas se probó sobre superficies de mesones de trabajo de la cocina del restaurante estudiantil de la Universidad Pedagógica y Tecnológica de Colombia - UPTC. Se siguió el proceder descrito por Carrascal, Páez y Burbano (1998), para evaluar el porcentaje de inhibición de microorganismos: bacilos gram negativos. Además, fue comparada su efectividad frente a dos conocidos desinfectantes comerciales: Mat- $98 \AA$ y Germigel ${ }^{\circledR}$.

\section{Diseño experimental}

La evaluación de la variable de respuesta: porcentaje de inhibición de bacilos gram negativos, se hizo en un modelo totalmente al azar, por tener unidades experimentales homogéneas, bajo el modelo $Y i j=m+t j+e i j$, con asignación de tratamientos en diseño balanceado multifactorial $3^{*} 32$ con dos factores de tratamiento: el factor $\mathrm{A}$, correspondiente a los tres compuestos evaluados (extracto de lulo, Germigel ${ }^{\circledR}$ y Mat-98®), y el factor B, que corresponde a tres tiempos $(2,5$ y 10 minutos) de contacto del extracto y los desinfectantes comerciales. Se determinaron los diferentes parámetros y se analizaron los resultados para los factores $A, B$ y $A B$ por anova, con un nivel de confianza del $95 \%$.

\section{RESULTADOS Y DISCUSIÓN}

\section{Materia seca}

Para el estudio se partió de $42,55 \% \pm 0,27 \%$ de pulpa fresca, de la cual se obtuvo el $4,06 \% \pm 0,97 \%$ de pulpa seca. Luego de tamizado el material se redujo en $1 \%$. El contenido de humedad y otras sustancias volátiles en el fruto alcanza el nivel del 95,64 - 96,8109\%, lo que incide en el bajo rendimiento del material seco y explica la drástica reducción de peso en el material seco del $38,49 \%$.

\section{Obtención del crudo de saponinas}

Los extractos hidroalcohólicos obtenidos por maceración de la pulpa seca y posterior concentración mostraron consistencia densa, color negro y olor a caramelo. El residuo obtenido con n-butanóico mostró consistencia pastosa y color rojo quemado, dando un rendimiento de 4,06\% $\pm 0,97$ con respecto al fruto fresco y de $79 \% \pm 1,2 \%$ con respecto al material seco.

\section{Identificación de saponinas}

Las pruebas cualitativas para confirmación de presencia de saponinas con resultados positivos se incluyen en la Tabla 1.

Tabla1. Ensayos químicos cualitativos en extracto de concentración hidroalcohólica

\begin{tabular}{|c|c|}
\hline Ensayo/extrac to & Concentración hidroalcohólica \\
\hline Espuma (sa poninas) & + \\
\hline Hemolisis (saponinas) & + \\
\hline Liebermann- Burchard (triterpenos y/o esteroides) & + \\
\hline Salko wski (triterpenos y/o esteroides) & + \\
\hline Antrona (sa poninas) & + \\
\hline Rosemheim (trite rpenos y/o este roides) & + \\
\hline Vain illina (sa pogeninas) & + \\
\hline
\end{tabular}


En la prueba de espuma, luego de agitar la muestra durante 2 minutos, se presentó una capa de espuma densa de $2,2 \mathrm{~cm}$, la cual se mantuvo estable por más de 6 horas. Debido a que esta prueba es solo presuntiva, se confirmó presencia de saponinas mediante la prueba de la hemólisis, aprovechando su sensibilidad de facilidad de discernimiento. Al adicionar el extracto sobre medio de cultivo agar-sangre, se presentó lisis evidente de los glóbulos rojos en el área afectada. Mediante la prueba de antrona se presentó un leve anillo azul en la interfase, el cual indica una reacción ligeramente positiva. Los ensayos más específicos para triterpenos y esteroides, tales como Liebermann-Burchard, Salkowski y Rosemheim, fueron francamente positivos. Para el primer caso, presentó formación de tres fases, y al final de la reacción se obtuvo una coloración negra, que indica la presencia de cantidades importantes del analito de interés. La coloración azul verdosa indica la presencia de saponinas de estructura esteroidal. Para el caso característico de sapogeninas (vainillina), la reacción con el extracto presentó una aparición de colores variables que indica una reacción positiva.

\section{Hidrólisis del crudo de saponinas}

A partir de $5 \mathrm{~g}$ de crudo de saponinas, por hidrólisis se obtuvieron 1,265 g de sapogeninas, y se logró un rendimiento del $24,1 \%$ con respecto al crudo. Respecto al material biológico presentó un rendimiento de $0,103 \% \pm 0,12 \%$; es relativamente bajo, ya que el fruto presenta un $39,846 \%$ de semillas, un $17,62 \%$ de corteza y solo un $42,55 \%$ de pulpa, que es donde se encuentra el analto de interés.

\section{Identificación de la sapogenina}

Para la identificación de las sapogeninas, se tuvieron como referencia los patrones de dos de ellas, teniendo como base los reportes bibliográficos, por ser las de mayor presencia en las especies vegetales de la familia analizada: diosgenina y hecogenina. Se realizó un análisis por espectroscopia infrarroja en equipo shimadzu prestige. En el espectro obtenido (ver espectro 1), se pueden observar claramente bandas pronunciadas y limpias, las cuales indican la alta pureza del producto obtenido. Se observan las siguientes bandas de vibración y tensión: grupo $\mathrm{OH}$ a una longitud de onda de 3304,06 cm-1; reporte teórico: 3500-2500 cm-1; grupos $\mathrm{CH}$ a 2927,94-2852,72 cm-1; reporte teórico: 2850-3095 cm-1; grupo carbonilo C=O a 1726,29 cm-1; reporte teórico: 1690$1760 \mathrm{~cm}-1$. Igualmente, se confirma la presencia de los grupos $\mathrm{CH}: \mathrm{CH}, \mathrm{CH} 2$ y CH3, en una longitud de onda de: $1606,92,1446,61$ y 1360,60 cm-1. Hacia 1202,66 cm-1 hay una tensión del grupo C-O, reporte teórico de: $1050-1300 \mathrm{~cm}-1$. Este espectro comparado con el patrón hecogenina de la biblioteca del equipo, tiene una similitud de un $99 \%$. El punto de fusión determinado del analito de interés fue $255-258^{\circ} \mathrm{C}$. Comparado con los patrones se asemeja más a la hecogenina, la cual tiene P.f de $258-262^{\circ} \mathrm{C}$. Se puede concluir que probablemente la sapogenina presente en mayor proporción en la especie Solanum marginatum es la hecogenina (Imagen 1).

\section{Imagen 1. Hecogenina}

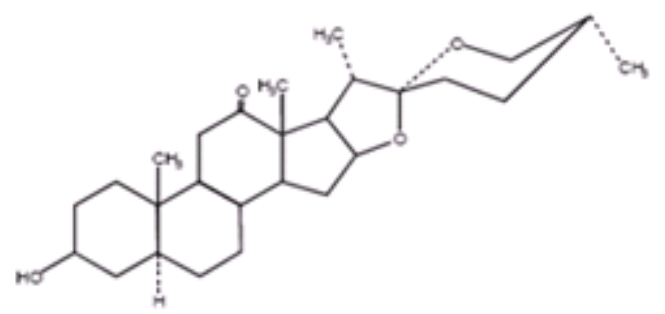

\section{Espectro 1. Sapogenina extraida de solanum marginatum}

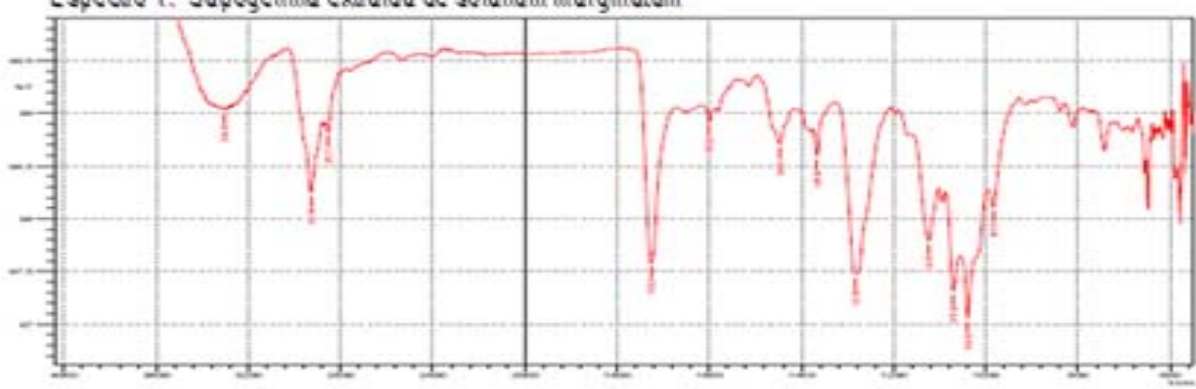

Evaluación del porcentaje de inhibición 
Los resultados de las pruebas de efectividad para medir el potencial sanitizante del extracto biodegradable, comparado con dos desinfectantes comerciales, son presentados en la Gráfica 1.

Grafica 1. Evaluación del porcentaje de inhibición.

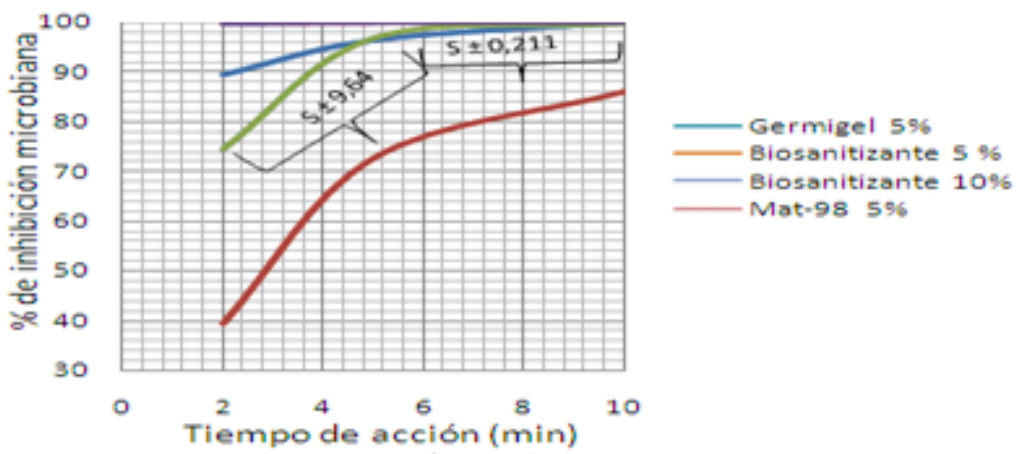

El porcentaje de inhibición sobre los microorganismos gram negativos depende de la concentración del biosanitizante y del tiempo de contacto sobre estos. En el extracto bajo, prueba que el mayor porcentaje de inhibición se presentó a partir de los 6 minutos a una concentración del 10\%, logrando un máximo de $99,58 \%$ de inhibición, tiempo a partir del cual la efectividad de todos los sanitizantes evaluados 
la poca diferencia de inhibición $(0,412 \%)$ con su particularidad de cuidar el entorno ambiental.

- El agente activo responsable del efecto sanitizante de la especie Solanum marginatum, según P.f. y I.R., corresponde a la sapogenina conocida como hecogenina.

- El rendimiento logrado de hecogenina en frutos de la especie Solanum marginatum fue de $0,103 \%$ con respecto al material biológico, y de $24,1 \%$ con respecto al material seco.

- Se recomienda probar la extracción del compuesto activo de esta especie en base húmeda, y comparar su rendimiento y eficiencia frente a la ruta utilizada en base seca.

\section{AGRADECIMIENTOS}

El autor del presente artículo expresa unos sinceros agradecimientos a las personas que colaboraron en el desarrollo del mismo: María Cristina Castellanos y Julia Reyes, docentes de Química; Luz Marina Lizarazo, docente de Microbiología; Edilma Díaz Tamayo, auxiliar de laboratorio de microbiología, y al personal administrativo del restaurante estudiantil de la Universidad Pedagógica y Tecnológica de Colombia, que me permitieron realizar los ensayos con el producto sanitizante.

\section{BIBLIOGRAFÍA}

- Carrascal, A. K.; Páez, A. \& Burbano, M. (2003). Manual de laboratorio: microbiología de alimentos. Colombia: CEJA.

- Fieser, L. (1959). "Saponinas esteroidales". Revista cubana de farmacia, 18.

- Herrera, A. \& Kecán, G. (1970). "Análisis fitoquímico y farmacológico del solanum marginatum". Rev. Colombiana ciencias químico-farmacéuticas, 3, 81-87.

- Hernández, R. R. (1997). Obtención de crudos de saponinas. Rev Cubana Med Milit, 26(1):55-62.

- Hostettman, K. \& Marston, A. (1995). "Chemistry and pharmacology of natural products". Joournald of natural products, 26. [New York].

- Martínez, M. A. (2001). Saponinas esteroides. Rev. Universitaria de Antioquia,52, 469-497. [Colombia].

- Wall, M. E. (1952). Saponinas esteroidales y triterpénicas. Anal Chem, 24.

- Sanabria, A. (1974). Alcaloides del solanum marginatum. Revista colombiana de ciencia químico-farmaceuticas, 38.

- Saponins. (1995). Studies in Natural Products Chemistry: Volumen 21, Parte 2 - Página 678. New York: Cambridge University Press.

- Segal, R.; Mansour, N. \& Saitschek, D. V. (1996). Effect of ester groups on the e haemolytic ofsome saponins and sapogenins. Journald biochem bharmacol, 15,1411-16.

1. Estudiante de Química de Alimentos. Semillero de investigación en Química Ambiental, Grupo GIQUA. Facultad de Ciencias. Universidad Pedagógica y Tecnológica de Colombia. Correo electrónico: fabrycy08@gmail.com .

2. Docente Escuela de Ciencias Químicas. Coordinadora Grupo de investigación en Química Ambiental, GIQUA. Facultad de Ciencias. Universidad Pedagógica y Tecnológica de Colombia.

Correo electrónico: mcristi.castellanos@gmail.com 